\title{
Functions of 1,25-dihydroxy vitamin D3,vitamin D3 receptor and interleukin-22 involved in pathogenesis of gout arthritis through altering metabolic pattern and inflammatory responses
}

\author{
Yuqi Chen ${ }^{1}$, Huiya Ma ${ }^{2}$, Youwei Du ${ }^{3}$, Jianjian Dong ${ }^{1}$, Chenkai Jin ${ }^{1}$, Lihui Tan ${ }^{1}$, Rong Wei ${ }^{\text {Corresp. } 1}$ \\ ${ }^{1}$ Department of Rheumatology and Immunology, the People's Hospital of Soochow New District, SuZhou, CHINA \\ 2 College of Chemistry and Pharmacy, Northwest A\&F University, Yangling, CHINA \\ ${ }^{3}$ State Key Laboratory of Crop Stress Biology for Arid Areas and College of Plant Protection, Northwest A\&F University, Yangling, China \\ Corresponding Author: Rong Wei \\ Email address: 952257684@qq.com
}

Background: Gouty arthritis (GA) is a common type of inflammatory arthritis. Recent studies demonstrated that 1,25-dihydroxy vitamin D3 (1,25(OH) 2 VD3) and vitamin D3 receptor (VD-R) play a protective role in acute inflammation, but interleukin-22(IL-22) promotes inflammation, especially for arthritis. However, our understanding of the responses of $1,25(\mathrm{OH}) 2$ VD3 and IL-22 to gout was still unclear. Presently, in-depth metabolomics, bioinformatics and clinical characteristics analyses were performed to elucidate the pathogenesis and valuable clinical indicators of gouty arthritis. Methods: Peripheral venous blood was taken for investigation. The levels of IL-22 and $1,25(\mathrm{OH})_{2} \mathrm{VD} 3$ were determined in patient's plasma via ELISA, and the mRNA levels of IL-22 and VD-R were measured via qRT-PCR. The interaction network of VD-R and IL22 were constructed by Search Tool for the Retrieval of Interacting Genes/Proteins (STRING), and the biological function of the related proteins were analyzed by Clusterprofiler Metabolomics were performed to decipher the metabolic variations of GA. Results: The levels of VD-R and

$1,25(\mathrm{OH}) 2$ VD3 were identified to be low. What's more, GA patients were reported to have high expression of IL-22. And IL-22 levels positively correlated with C-reactiveprotein (CRP) serum levels in the bivariate correlation analysis, whereas the level of 1,25(OH) 2VD3 negatively correlated with that of CRP. GO and KEGG analyses revealed that IL-22 and $1,25(\mathrm{OH}) 2$ VD3 were involved in stress immunity and inflammatory responses. These pathways are known to play a role in GA pathogenesis. Meanwhile, the metabolic profiles of GA serum showed that the increase in various amino acids and uric acid are involved in GA pathogenesis. Importantly, VD-R and IL22 closely correlated with the level of key metabolites uric acid, whose increase promoted the occurrence of GA. Conclusion: GA 
patients have low levels of VD-R and 1,25(OH) 2 VD3, and high levels of IL-22 together with various amino acids and uric acid. The levels of IL-22 and 1,25(OH) 2VD3 were positively and negatively correlated with C-reactive protein (CRP) serum levels, respectively. Both IL-22 and 1,25(OH) 2 VD3 functioned in GA-related immune and inflammatory responses, and closely correlated with the level of GA-related uric acid. Overall, IL-22, VD-R and 1,25(OH) 2 VD3 play functionally important roles in inflammatory responses and are relevant to gout pathogenesis. 
1

2

3

4

5

6

7

8

9

\section{Functions of 1,25-dihydroxy vitamin D3, vitamin D3 receptor and interleukin-22}

involved in pathogenesis of gout arthritis through

\section{altering metabolic pattern and inflammatory responses}

Yuqi Chen ${ }^{1}$, Huiya $\mathrm{Ma}^{2}$, Youwei $\mathrm{Du}^{3}$, Jianjian Dong ${ }^{1}$, Chenkai Jin ${ }^{1}$, Lihui Tan ${ }^{1}$, Rong Wei ${ }^{1 *}$

${ }^{1}$ Department of Rheumatology and Immunology, the People's Hospital of Suzhou New District, 215000,

Suzhou, China

${ }^{2}$ College of Chemistry and Pharmacy, Northwest A\&F University, No.22 Xinong Road, Yangling 712100,

Shaanxi, China

${ }^{3}$ State Key Laboratory of Crop Stress Biology for Arid Areas and College of Plant Protection,

Northwest A\&F University, Yangling, 712100, Shaanxi, China

Corresponding author: Rong Wei, Department of Rheumatology and Immunology, the People's Hospital of

Suzhou New District, 215000, Suzhou, China. Email: 952257684@qq.com

Key words: gouty arthritis; uric acid; interleukin-22; 1,25-dihydroxy vitamin D3; vitamin D3 receptor 


\section{Abstract}

Background: Gouty arthritis (GA) is a common type of inflammatory arthritis. Recent studies demonstrated that 1,25-dihydroxy vitamin D3 $(1,25(\mathrm{OH}) 2 \mathrm{VD} 3)$ and vitamin $\mathrm{D} 3$ receptor (VD-R) play a protective role in acute inflammation, but interleukin-22(IL-22) promotes inflammation, especially for arthritis. However, our understanding of the responses of 1,25(OH) 2VD3 and IL-22 to gout was still unclear. Presently, in-depth metabolomics, bioinformatics and clinical characteristics analyses were performed to elucidate the pathogenesis and valuable clinical indicators of gouty arthritis.

Methods: Peripheral venous blood was taken for investigation. The levels of IL-22 and 1,25(OH $)_{2}$ VD3 were determined in patient's plasma via ELISA, and the mRNA levels of IL-22 and VD-R were measured via qRTPCR. The interaction network of VD-R and IL22 were constructed by Search Tool for the Retrieval of Interacting Genes/Proteins (STRING), and the biological function of the related proteins were analyzed by Clusterprofiler Metabolomics were performed to decipher the metabolic variations of GA.

Results: The levels of VD-R and 1,25(OH) 2 VD3 were identified to be low. What' s more, GA patients were reported to have high expression of IL-22. And IL-22 levels positively correlated with C-reactive protein (CRP) serum levels in the bivariate correlation analysis, whereas the level of $1,25(\mathrm{OH}) 2 \mathrm{VD} 3$ negatively correlated with that of CRP. GO and KEGG analyses revealed that IL-22 and 1,25(OH) 2 VD3 were involved in stress immunity and inflammatory responses. These pathways are known to play a role in GA pathogenesis. Meanwhile, the metabolic profiles of GA serum showed that the increase in various amino acids and uric acid are involved in GA pathogenesis. Importantly, VD-R and IL22 closely correlated with the level of key metabolites uric acid, whose increase promoted the occurrence of GA. 
38 Conclusion: GA patients have low levels of VD-R and 1,25(OH) 2 VD3, and high levels of IL-22 together

with various amino acids and uric acid. The levels of IL-22 and 1,25(OH) $2 \mathrm{VD} 3$ were positively and negatively correlated with C-reactive protein (CRP) serum levels, respectively. Both IL-22 and 1,25(OH) 2

VD3 functioned in GA-related immune and inflammatory responses, and closely correlated with the level of GA-related uric acid. Overall, IL-22, VD-R and 1,25(OH) 2 VD3 play functionally important roles in inflammatory responses and are relevant to gout pathogenesis.

\section{Introduction}

Gout is a major public health problem impacting human health(Stack et al. 2019). Currently, the prevalence of gout was higher ranging from 1-4\%, and patients with gout suffer from dramatic joint inflammation and excruciating arthritic pain, which profoundly reduced the patients' life quality(Singh \& Gaffo 2020); (Yin et al. 2020). Meanwhile, comorbidities such as hypertension, stroke, and heart disease, chronic kidney disease are common in people with gout and complicate its management and disease outcomes (Kuwabara et al. 2017). It is a complex disorder caused by the deposition of monosodium urate (MSU) crystals in joints and soft tissues(Zhu et al. 2018). MSU stimulates the activation of the innate immune system and subsequently releases an array of inflammatory mediators(Nakayama 2018; Yin et al. 2020). These inflammatory factors trigger a strong inflammatory response in joints and periarticular tissues (Richette \& Bardin 2010). However, the exact pathogenesis of gout is yet to be elucidated.

Elevated levels of specific pro-inflammatory cytokines are known to trigger highly reactive inflammatory infections. Intriguingly, 1,25(OH) 2 VD3 can inhibit inflammation and is commonly found in a range of immune cells. This suggests it plays a key role in the immune system(Ho et al. 2020). Adding to this hypothesis, Wen $\mathrm{H}$ indicated that 1,25(OH) 2 VD3 may play as an immune modulating agent to suppress Th17 
59

60

61

62

63

64

65

66

67

68

69

70

71

72

73

74

75

76

77

78

79

cell cytokines, such as IL-22(Wen et al. 2015). In this study, we hypothesize that IL-22, is correlated with the occurrence and development of gout. We also hypothesized that 1,25(OH) 2 VD3 plays an anti-inflammatory role in gouty arthritis and can even suppress episode of gout.

This study investigated changes in the serum levels of 1,25(OH) 2 VD3 and IL-22 in the peripheral blood of gout arthritis patients. Correlations between serum levels and sedimentation rates of CRP, serum uric acid, creatinine, triglycerides, cholesterol and platelets with 1,25(OH) 2 VD3, and IL-22 were subsequently investigated. Finally, the biological functions of 1,25(OH) 2 VD3 and IL-22 in gout pathogenesis were further explored via bioinformatics analysis, and the detailed variations in metabolic pattern of GA were displayed by metabolomics profiles. These findings provide valuable insight into the mechanisms underpinning gout pathogenesis and provide more ideas for the treatment of gouty arthritis.

\section{Methods}

\subsection{Gout patients recruitment}

A total of 58 gout outpatients were recruited from the Rheumatism and Immunology Department of the People's Hospital of Suzhou New District, China. These patients were recruited between March 2019 and September 2019. To be included in the study, patients had to fit into the criteria defined by the American Rheumatism Association (now named the American College of Rheumatology (ACR)) in 1977(Wallace et al. 1977) and the ACR/European League Against Rheumatism (EULAR) in 2015(Neogi et al. 2015). Patients were categorized into two groups: acute (in the last week, with joint swelling and pain, and increased white blood cell or erythrocyte sedimentation rate, $\mathrm{n}=30$ ) and non-acute (No joint swelling and pain occurred in the 
80

81

82

83

84

last month, acute joint swelling and pain occurred in the past, and no colchicine, prednisone, non-steroidal anti-inflammatory drugs and urate-lowering therapy was given, $\mathrm{n}=28$ ) GA.

Patients with secondary gout as a result of drugs, chemoradiotherapy, malignant tumor, endocrine and kidney diseases were excluded from the study. Twenty healthy physical examining persons were recruited as healthy controls who were lacking hyperuricemia and metabolic syndromes as well as other chronic diseases. Participants provided fasting morning venous blood samples and the specific operation was completed by nurses. Participants were matched for age and sex. Written informed consent was obtained from all patients at the time of admission. The People's Hospital of Suzhou New District granted Ethical approval to carry out the study within its facilities (Ethical Application Ref: 2018-006).

\subsection{Laboratory indexes}

Laboratory indexes were determined in the laboratory of Suzhou High-Tech Zone at the People's Hospital. Blood cell analysis was performed with the blood cell analyzer LH750 (Beckman, US). Analyses including erythrocyte sedimentation rate (ESR), CRP, uric acid, urea, and creatinine were performed with a DxC800 automatic biochemical analyzer (Beckman, US).

\subsection{Quantitative PCR (qPCR)}

Whole peripheral blood samples were used for total cellular RNA isolation. Total RNA was extracted using a standard TRIZOL method following(Qin et al. 2013) and cDNA was generated using the PrimeScript

RT Reagent kit (Takara). IL-22 and VD-R expression was quantified via qPCR using a LightCycler 480 and SYBR Green I Master Mix (Roche). Expression levels were normalized to glyceraldehyde 3-phosphate 
101

102

103

104

105

106

107

108

109

110

111

112

113

114

115

116

117

118

119

120

121

dehydrogenase (GAPDH). Water was used as a negative control lacking template. A standard curve was

generated for each assay. Amplification efficiency was calculated based on the mRNA standard curve. Assays were performed in duplicate. The average used in statistical analyses.

\subsection{Enzyme-linked immunosorbent assay (ELISA)}

Peripheral blood was collected in heparin anticoagulant tubes. Plasma was obtained via centrifugation at

3000 RPM for 15 minutes. Prior to examination, plasma was stored at $-20^{\circ} \mathrm{C}$. Plasma levels of IL-22 and

1,25(OH)2VD3 were determined using a quantitative sandwich technique, for which ELISA kits (eBioscience,

US) were used according to the manufacturer's instructions.

\subsection{Enrichment and protein-protein interaction network analysis:}

Gene ontology (GO) enrichment analysis of differentially expressed genes was performed using the ClusterProfiler R package. GO terms with a corrected $p>0.05$ were considered enriched by differentially expressed genes. The Kyoto Encyclopedia of Genes and Genomes (KEGG) database was subsequently used.

This database contains a wealth of understanding of molecular-level information, including large-scale molecular datasets generated via genome sequencing and other high throughput technologies

(http://www.genome.jp/kegg/)

\subsection{Metabolomics analysis:}

To investigate the potential variations in the metabolisms of patients, the serum of GA patients and

healthy controls were collected for a pilot of metabolomics analysis. To identified the most relevant metabolic 
122

123

124

changes involved in the pathogenesis of GA, we used pooled serum extracts from five replicated individuals as one sample. Detailed steps of metabolomics analysis are as follows: $50 \pm 1 \mathrm{ml}$ sample from GA patients $(\mathrm{N}=15)$ and healthy control $(\mathrm{N}=15)$ were extracted with pre-cold extraction mixture (methanol/chloroform $(\mathrm{v}: \mathrm{v})=3: 1)$ with $10 \mu \mathrm{L}$ internal standard (adonitol), and samples were extracted with ultrasonication for 5 min in ice water.

Every five extracts were mixed into one pooled sample. In total, three pooled replicates of GA group or healthy group were used for the pilot metabolomics. After evaporation in a vacuum concentrator, $40 \mu \mathrm{L}$ of Methoxyamination hydrochloride $\left(20 \mathrm{mg} / \mathrm{mL}\right.$ in pyridine) was added and then incubated at $80{ }^{\circ} \mathrm{C}$, then derivatized by $60 \mu \mathrm{L}$ of BSTFA regent $(1 \% \mathrm{TMCS}, \mathrm{v} / \mathrm{v})$ at $70{ }^{\circ} \mathrm{C}$ for $1.5 \mathrm{~h}$.

All samples were then analyzed by gas chromatograph using an Agilent 7890 with a time-of-flight mass spectrometer (GC-TOF-MS) and DB-5MS capillary column. $1 \mu \mathrm{L}$ aliquot of sample was injected in splitless mode. Helium was used as the carrier gas with $1 \mathrm{~mL} \min ^{-1}$ flow rate. The energy was $-70 \mathrm{eV}$ in electron impact mode. The mass spectrometry data were acquired in full-scan mode with the $\mathrm{m} / \mathrm{z}$ range of 50-500 at a rate of 12.5 spectra per second.

\subsection{Raw Data Preprocessing.}

Raw data analysis was finished with Chroma TOF (V 4.3x, LECO) software and LECO-Fiehn Rtx5

database was used for metabolite identification with an in-house database. The noise and deviation values were removed by filtering individual peaks and the interquartile range, respectively. Only the peak area data with a hollow value not more than $50 \%$ in all groups were retained, then the missing value was simulated by $1 / 2$ of the minimum value. And the data were normalized by an internal standard (IS). The data variation were

performed by R packages XCMS software. The Principle Component Analysis (PCA) and pathway enrichment analysis were performed by Metaboanalyst 3.0. The PCA showed the metabolic variations of GA patients. 
143 PCA was performed on the three-dimensional metabolic data involving the metabolite name, sample name and

144 normalized peak area. The data were further treated through mean centering and unit variance scaling. The

145 PCA plots was generated to interpret cluster separation. And the therapy $\mathrm{P}<0.05$ and FC $>2$ were used for

146 identification of significantly differential metabolites.

$148 \quad$ 2.8. Statistical analysis

Statistical analyses were performed using SPSS Statistics Version 21 (IBM). The groups were tested

using two-sided $t$ test or ANOVA for continuous variables and the Chi-squared statistic for categorical

variables. A two-sided $p<0.05$ was used to denote statistical significance. Baseline characteristics were

presented as mean (with an associated standard deviation [SD]) for continuous variables. For dichotomous

variables, proportions (\%) were used. Mann-Whitney U tests were used for non-normally distributed variables.

A Chi -square test or Fisher's exact test were used for categorical variables. Pearson or Spearman's rank correlation tests were used depending on the data distribution.

\section{Results}

\subsection{Patient characteristics}

The clinical characteristics of recruited gout patients were documented and analyzed. These

characteristics included age, body mass index (BMI), uric acid and creatinine levels, platelet levels,

erythrocyte sedimentation rate (ESR) and C-reactive protein (CRP) levels, cholesterin levels, triglyceride 
164

165

166

167

168

169

170

171

172

173

174

175

176

177

178

179

180

181

182

183

184

patients, and 20 healthy controls. Participants were age- and sex-matched. It's important to note that the BMIs of patients in the AGA and NAGA groups were significantly higher than those in $\operatorname{HCs}(p<0.05)$.

This supports the notion that obesity may be a risk factor for gout. Besides, Creatinine levels are higher in patients with gout than in normal subjects, reflecting the fact that gout can impair kidney function. What's more, UA, blood glucose, and ESR were all significantly higher in AG patients than in HC patients. In addition, in AGA patients, CRP levels were found to be highly upregulated in comparison to the NAGA and $\mathrm{HC}$ groups. There were no differences in the disease duration, hypertension rates, and diabetes rates in the NAGA patients vs. the AGA patients $(p>0.05)$ (Table 1).

AGA: acute gouty; BMI: body mass index; CRP: C-reactive protein;

ESR: erythrocyte sedimentation rate; HC: healthy controls; NAGA: non-acute gouty arthritis; UA: uric acid; $* p<0.05$, between AGA patients vs. HC; \# $p<0.05$, between NAGA patients vs. HC; $\triangle p<0.05$, between NAGA patients vs. AGA patients;

\subsection{IL-22, VD-R and 1,25(OH) ${ }_{2} V D 3$ levels closely correlated with gout pathogenesis}

IL-22 is a highly important cytokine and performs various anti-inflammatory and pro-inflammatory functions in vivo. In this study, qRT-PCR was performed to investigate the expression levels of IL-22. In comparison to the HC group, IL-22 expression was highly upregulated in the AGA and NAGA groups. On another note, as the receptor for vitamin D, VD-R plays a key role in regulation of the immune system (Medrano et al. 2018). Remarkably, this study reported that VD-R expression levels were dramatically downregulated in the AGA and NAGA groups (Figure1). Subsequent, ELISA analysis and qRT-PCR experimentation revealed a consensus expression level of IL-22 and VD3. What's more, ELISA indicated that IL-22 protein expression was significantly upregulated in both the AGA and NAGA groups. In addition, 
$1,25(\mathrm{OH})_{2} \mathrm{VD} 3$ protein expression was also identified to be significantly decreased in the AGA group by

ELISA assay. There was no clear difference between the NAGA and $\mathrm{HC}$ group in terms of $1,25(\mathrm{OH})_{2} \mathrm{VD} 3$

protein expression levels (Figure1).

A: Serum 1,25(OH)2VD3 levels in HC, AGA, and NAGA groups (mean $\pm \mathrm{SD}$ ), $p$-value $<0.01$; B: Serum IL22 levels in HC, AGA, and NAGA groups (mean $\pm \mathrm{SD}$ ), $p$-value $<0.01$; VD-R(C)and IL-22(D) mRNA levels in serum, Data represent mean \pm SD. $p$-value $<0.01$;

\subsection{Levels of IL-22 and VD-R closely correlated with GA-related metabolites}

In order to further identified the potential metabolic variations of GA patients, untargeted metabolomics was performed. This pilot of metabolomics analysis was performed on the serum from both HC and GA patients. Three replicates were performed for each group. Principal components analysis (PCA) was performed on the metabolic profiles of both the HC and GA patients. This analysis showed that both groups had a distinct profile. This showed that there is measurable variation in metabolic profiles of $\mathrm{HC}$ and GA patients (Figure 2C). Furthermore, we constructed a coexpression network on IL22, VDR and metabolites from metabolomics.

We found various metabolites involved in Aminoacyl-tRNA biosynthesis, Pantothenate and CoA biosynthesis, Purine metabolism, Phenylalanine, tyrosine and tryptophan biosynthesis, Arginine biosynthesis, Valine, leucine and isoleucine biosynthesis, beta-Alanine metabolism and Phenylalanine metabolism were closely correlated with IL22 and VDR (Figure 2A; Table 2). We noted that six metabolites including 3-

Hydroxyanthranilic acid, Palatinose, sulfuric acid, serine, 2,6-Diaminopimelic acid, Phytol and uric acid were positively correlated with IL22, whereas their correlation with VDR were negative (Figure 2A). The level of these metabolites were higher in GA patients than that in HC (Figure 2B). Of note, metabolic alterations 
206

207

208

209

210

211

212

213

214

215

216

217

performed various functions in the pathogenesis of GA(Hoque et al. 2020). Subsequently, 446 metabolites were identified in the serum. Out of these metabolites, the levels of 147 metabolites were highly increased in serum of GA patients. The levels of 33 metabolites were decreased in the serum of GA patients (Figure 2D). Subsequent pathway enrichment analysis, using the KEGG database, was performed on the 147 metabolites upregulated in GA patients. This analysis identified 45 pathways, 9 pathways of which were significantly enriched (Figure 2E). This included the following pathways: aminoacyl-tRNA biosynthesis; arginine biosynthesis; pantothenate and CoA biosynthesis; alanine, aspartate and glutamate metabolism; phenylalanine, tyrosine and tryptophan biosynthesis; glycine, serine and threonine metabolism; galactose metabolism; glutathione metabolism and valine; leucine and isoleucine biosynthesis, revealing the influence of these metabolic alterations which correlated with IL22 and VDR in the pathogenesis of GA. In this study, a variety of amino acid-related pathways were significantly activated in GA patients. This indicates that a high level of amino acids are involved in GA pathogenesis. Notably, research has shown that elevated levels of proteinuria is indicative of arthritis(Luo et al. 2018; Teumer et al. 2019). In agreement, this study identified that serum uric acid levels of GA patients were 2.8-fold higher than that in the $\mathrm{HC}$ group (Figure 2B). Uric acid is known for its inductive function in pathogenesis of GA(Huang et al. 2020). Remarkably, levels of IL-22 and VD-R closely correlated with the uric acid levels in the serum of both $\mathrm{HC}$ and GA patients. Thus, We concluded that IL22 and VD-R were closely correlated with the GA-related metabolic alterations, supporting their functions in the pathogenesis of GA.

A: Coexpression network between IL22, VDR and metabolic signature; Negative and positive relationship were shown represented by red and blue line, respectively; B: Relative level of metabolites which were closely associated with both IL22 and VDR; C: PCA plots of the metabolomics patterns of the GA and HC groups; D: 
227 Volcano plots displayed differential metabolites in the metabolomics data of the GA and HC groups; E:

228 Pathway enrichment analysis of the upregulated metabolites of GA patients.

\subsection{IL-22 and 1,25 $(\mathrm{OH})_{2} \mathrm{VD} 3$ levels in serum closely correlated with $\mathrm{CRP}$ levels}

As shown in Figure 3, the level of IL-22 in the serum positively correlated with CRP levels $(\mathrm{r}=0.32, p<$

0.05). In addition, $1,25(\mathrm{OH})_{2} \mathrm{VD} 3$ levels negatively correlated with CRP levels $(\mathrm{r}=-0.40, p<0.01)$ (Figure 3 ).

233

234

235

236

237

238

239

240

241

242

243

244

245

246

247

CRP is a non-specific inflammatory marker. Interestingly, IL-22 levels in the serum positively correlated with

CRP levels. However, the serum levels of $1,25(\mathrm{OH})_{2} \mathrm{D} 3$ negatively correlated with the serum CRP levels.

Correlation analysis was performed to confirm the correlations between the levels of IL-22, $1,25(\mathrm{OH})_{2} \mathrm{D} 3$ and other measurements such as platelets, ESR. No significant correlations were identified between these indicators. Similarly, the correlation between CRP and IL-22 and VD3 are not similar for all 3 groups of participants. These findings suggested that $1,25(\mathrm{OH})_{2} \mathrm{VD} 3$ and IL-22 are associated with inflammatory expression in gout arthritis.

\subsection{IL-22 and VD-R are imperative in the inflammatory response network}

In order to further investigate the function of IL-22 and VD-R in the pathogenesis of gout, a complex protein-protein interaction network was constructed using Search Tool for the STRING. The created network showed that various genes were involved in inflammatory response. These genes included STA3, MED25, MED22, STAT1, CREB1, HIF1, and IL-10 (Figure 4D). These interactions indicated that IL-22 and VD-R could in fact further influence protein functions associated with the inflammatory response. The topology of this complex network was subsequently analyzed. This analysis showed that IL-22 and VD-R were at the core 
248 of the network. This suggests important roles for both IL-22 and VD-R in the regulation inflammatory

249 responses (Figure 4D).

250 Furthermore, GO enrichment analysis was performed on the identified regulatory network. This analysis

251 showed that these proteins were involved in various terms associated with the cellular component category.

252 This included various components: transcription factor complex, RNA polymerase II transcription factor complex, protein-containing complex, nucleus, nucleoplasm part, and nuclear transcription factor complex

(Figure 4A). For the molecular function category, protein functions were mainly involved in vitamin D

receptor binding, transcription regulator activity, transcription factor binding, thyroid hormone receptor

noted that various terms associated with biological progress were overrepresented. This included transcription,

DNA-templates, transcription initiation from the RNA polymerase II promoter, RNA metabolic process,

(Figure 4E). In summary, these results agreed that IL-22 and VD-R play functionally important roles in the 
269

270

271

272

273

274

275

276

277

278

279

280

281

282

283

284

285

286

287

288

289

analysis on the regulatory network of IL-22 and VD-R; C: Biological Progress analysis on the regulatory

network of IL-22 and VD-R; D: The regulatory network of IL-22 and VD-R; E:Pathway enrichment analysis on VD-R and IL-22.

\section{Discussion}

Gout arthritis is an auto-inflammatory disease(Salehzadeh et al. 2019). The acute onset of gout is an inflammatory process induced by urate crystals. These crystals manifest as inflammatory arthritis and cause painful disability. Various researchers have proposed that the gout arthritis is closely linked with various inflammatory reactions, including NLRP3, IL-1 and IL-22(Cabau et al. 2020; Luo et al. 2017). However, the role of IL-22 and VD3 in gout pathogenesis is yet to be elucidated. In this study, GO and KEGG pathway analyses identified important roles of both IL-22 and 1,25(OH) 2 VD3 in stress immunity as well as inflammatory responses. These processes are known to be associated with gout arthritis pathogenesis.

Remarkably, the levels of IL-22 were shown to be higher in patients with gouty arthritis in either the acute phase or non-acute phase in comparison to healthy controls. What's more, decreased levels of VD-R mRNA and $1,25(\mathrm{OH})_{2}$ VD3 in both AGA and NAGA groups were verified via ELISA and qRT-PCR.

Furthermore, the levels of IL-22 and VD3 correlated with that of CRP in gout patients. Importantly, we found that uric acid highly accumulated in GA patients, and the levels of VD-R and IL22 closely correlated with the level of uric acid, supporting the imperative roles of VD-R and IL22 in the pathogenesis of GA. In summary, this study reported the involvement of IL-22 and VD3 in inflammation associated with gout pathogenesis.

These results suggest a pivotal role of IL-22 and VD3 in mediating gout inflammation. These findings provide a novel treatment strategy for gout. 
to its specific function in vivo. Remarkably, this study showed that mRNA expression levels of IL-22 were

292

293

294

295

296

297

298

299

300

301

302

303

304

305

306

307

308

309

310

higher in the acute and remission phases of gout. This indicates that IL-22 was involved in the pathogenesis of gouty arthritis. Previous research has proven the role of IL-22 in the regulation of inflammatory responses associated with inflammatory diseases by binding to the IL-22 receptor. This receptor is transmembrane in nature, and is composed of two subunits (IL-22R1 and IL-10R2)(Lu et al. 2015; Perusina Lanfranca et al. 2016). Notably, IL-22 is characterized as a two-faced cytokine. This means it performs not only deleterious roles but also protective roles(Huan et al. 2016). It is also an important driver of autoimmune diseases and is involved in the pathogenesis of arthritis(Yang \& Zheng 2014). A range of studies have shown that patients with rheumatoid arthritis and psoriatic arthritis frequently have abnormal IL-22 expression(Ezeonyeji et al. 2017; Miyazaki et al. 2018). IL-22 promoted inflammatory response and osteoclast formation in rheumatoid arthritis via the promotion of fibroblast-like synoviocytes proliferation and monocyte chemoattractant protein (MCP) expression(Li et al. 2019). Marijnissen et al. reported IL-22 and IL-22 receptor levels to be increased in synovial tissue during inflammation(Marijnissen et al. 2011). On another note, IL-22 promoted the expression of IFN- $\gamma$, thereby inhibiting intestinal coronavirus replication(Xue et al. 2017). IL-22 and IFN- $\gamma$ have been shown to suppress pulmonary inflammation in pneumonia caused by Pseudomonas aeruginosa (Broquet et al. 2020). The therapeutic benefits of IL-22 pathway activation have been demonstrated in experimental colitis(Lamas et al. 2016). In this study, levels of IL-22 in plasma were increased in both acute and chronic gout patients. These levels correlated positively with CRP levels. This suggests the imperative role of IL-22 in the pathogenesis of gout arthritis.

As an active vitamin $\mathrm{D}$ in vivo, $1,25(\mathrm{OH})_{2} \mathrm{VD} 3$ plays role in maintaining the balance of calcium and

Peer] reviewing PDF | (2021:07:63744:2:1:NEW 3 Nov 2021) 
311

312

phosphorus various biological processes by binding to VD-R. Detailed studies have shown that $1,25(\mathrm{OH})_{2}$ VD 3

plays an important role in immune regulation, anti-inflammation, and anti-infection(Holick 2014). In this study, we reported that the levels of VD-R mRNA and protein were significantly decreased in both the AGA and NAGA group. This suggests that VD-R may play a negative regulatory role in the inflammatory process of gout arthritis. The decrease in $1,25(\mathrm{OH})_{2} \mathrm{VD} 3$ levels in serum are potentially caused by high uric acid levels(Chen et al. 2014). A study has described that $1,25(\mathrm{OH})_{2} \mathrm{VD} 3$ effectively inhibited Th17 cell differentiation in vitro and regulated the immune response of $\mathrm{T}$ cells. The study reported that $1,25(\mathrm{OH})_{2} \mathrm{VD} 3$ treatment could cause decreases in the levels of IL-17A, IL-22 and IFN- $\gamma(\mathrm{Gu}$ et al. 2017). What's more, $1,25(\mathrm{OH})_{2} \mathrm{VD} 3$ promoted the formation of STAT1-VD-R complexes in monocytes. Interestingly, $1,25(\mathrm{OH})_{2} \mathrm{VD} 3$ significantly reduced the transcriptional activity of VD-R, but enhanced the transcription of STAT1. Thus, it can be hypothesized that correlations between the VD-R and STAT signaling pathways exist, and that the anti-inflammatory effects of $1,25(\mathrm{OH})_{2} \mathrm{VD} 3$ may be related to the effects of vitamin $\mathrm{D}$ on the JAK/STAT pathway. Another study found that $1,25(\mathrm{OH})_{2} \mathrm{VD} 3$ can in fact control the expression of VD-R, signal transducers, and transcriptional activator 5 (STAT5) via the regulation of signal transduction pathways(Yang et al. 2015). KEGG pathway analyses showed that VD-R and IL-22 mRNA were mainly concentrated in multiple signal transduction pathways such as the JAK/STAT signaling pathway in tuberculosis, prostate cancer, and inflammatory bowel disease. VD-R can combine with STAT3 to inhibit the JAK/STAT signaling transduction pathway(Zhang et al. 2020). Research has also proven that $1,25(\mathrm{OH})_{2} \mathrm{D} 3$ can be expressed via the promotion of STAT5 and its phosphorylation(Gu et al. 2017). This can in turn promote Th17 cells specific cytokine IL-17 as well as the expression and secretion of IL-22. Andoh et al. reported that IL-22 raised IL-6, IL-8, IL-11, and other inflammatory mediators and chemokine expression by 
332

activating SEMF nf-kappa B cells, JAK-2/STAT and AP-1-3 signaling pathways(Andoh et al. 2005). Thus exerting a pro-inflammatory effect.

As a non-specific inflammatory marker, CRP can bind to a variety of eukaryotic and prokaryotic pathogens, facilitating complement activation through the classical pathway(Pepys \& Hirschfield 2003). This leads to immune activation, lymphocyte infiltration, immune molecules consumption, and widespread inflammation. Levels of IL-22 positively correlated with serum CRP levels, which confirmed that IL-22 was involved in the inflammatory response in the pathogenesis of gout arthritis. Serum 1,25(OH)2D3 levels negatively correlated with serum CRP levels. This indicated that VD3 played a protective role in gout. In addition, functional enrichment and KEGG signaling pathway analyses of target genes predicted the molecular regulatory networks related to disease occurrence. In parallel, we detailedly depicted the landscape of metabolic alterations between gout patients and normal human that the levels of various amino acids and uric acid associated with the gout pathogenesis were higher in gout patients. What's more, serum VD-R and IL-22 were increased in patients and may be involved in the pathogenesis of gout arthritis via influencing the uric acid level of gout patients that both VD-R and IL-22 closely correlated with the level of uric acid of GA patients. This goes some way to elucidate the molecular regulatory network controlling gout arthritis pathogenesis.

However, it is important to note that there are limitations to the interpretation of these results due to the small sample size. And the metabolomics analyses is also a pilot of concept study. Thus, some conclusions relevant to metabolic variations of GA patients were hypothetical at this stage. However, our study provided multiple evidences that the levels of $1,25(\mathrm{OH})_{2} \mathrm{VD} 3$ and IL-22 were reported to be abnormal in the serum of patients with gout arthritis, and both of them involved in the pathogenesis of gout arthritis. And the specific 
353

354

355

356

357

358

359

360

361

362

363

364

365

366

367

368

369

370

371

372

373

374

375

376

377

378

379

380

381

382

383

384

mechanism underpinning this remain unclear. Therefore, further studies are required to confirm the

mechanisms via which $1,25(\mathrm{OH})_{2} \mathrm{VD}_{3}$ affects the secretion of IL-22 in gout.

\section{Acknowledgements}

The authors thank the Key Project of Scientific Innovation Fund Grant of People's Hospital of Suzhou New

District for supporting this work. Thanks are due to Dr Ma for assistance with the experiments and to D. He for valuable discussion.

\section{References}

Andoh A, Zhang Z, Inatomi O, Fujino S, Deguchi Y, Araki Y, Tsujikawa T, Kitoh K, Kim-Mitsuyama S, Takayanagi A, Shimizu N, and Fujiyama Y. 2005. Interleukin-22, a member of the IL-10 subfamily, induces inflammatory responses in colonic subepithelial myofibroblasts. Gastroenterology 129:969-984. 10.1053/j.gastro.2005.06.071

Broquet A, Besbes A, Martin J, Jacqueline C, Vourc'h M, Roquilly A, Caillon J, Josien R, and Asehnoune K. 2020. Interleukin-22 regulates interferon lambda expression in a mice model of pseudomonas aeruginosa pneumonia. Mol Immunol 118:52-59. 10.1016/j.molimm.2019.12.003

Cabau G, Crisan TO, Kluck V, Popp RA, and Joosten LAB. 2020. Urate-induced immune programming: Consequences for gouty arthritis and hyperuricemia. Immunol Rev 294:92-105. 10.1111/imr.12833

Chen W, Roncal-Jimenez C, Lanaspa M, Gerard S, Chonchol M, Johnson RJ, and Jalal D. 2014. Uric acid suppresses 1 alpha hydroxylase in vitro and in vivo. Metabolism 63:150-160. 10.1016/j.metabol.2013.09.018

Ezeonyeji A, Baldwin H, Vukmanovic-Stejic M, and Ehrenstein MR. 2017. CD4 T-Cell Dysregulation in Psoriatic Arthritis Reveals a Regulatory Role for IL-22. Front Immunol 8:1403. 10.3389/fimmu.2017.01403

Gu L, Xu Q, and Cao H. 2017. 1,25(OH)2D3 Protects Liver Fibrosis Through Decreasing the Generation of TH17 Cells. Med Sci Monit 23:2049-2058. 10.12659/msm.904271

Ho J, Chan H, Liang Y, Liu X, Zhang L, Li Q, Zhang Y, Zeng J, Ugwu FN, Ho IHT, Hu W, Yau JCW, Wong SH, Wong WT, Ling L, Cho CH, Gallo RL, Gin T, Tse G, Yu J, Chan MTV, Leung CCH, and Wu WKK. 2020. Cathelicidin preserves intestinal barrier function in polymicrobial sepsis. Crit Care 24:47. 10.1186/s13054-020-2754-5

Holick MF. 2014. Sunlight, ultraviolet radiation, vitamin D and skin cancer: how much sunlight do we need? Adv Exp Med Biol 810:1-16.

Hoque KM, Dixon EE, Lewis RM, Allan J, Gamble GD, Phipps-Green AJ, Halperin Kuhns VL, Horne AM, Stamp LK, Merriman TR, Dalbeth N, and Woodward OM. 2020. The ABCG2 Q141K hyperuricemia and gout associated variant illuminates the physiology of human urate excretion. Nat Commun 11:2767.

Peer] reviewing PDF | (2021:07:63744:2:1:NEW 3 Nov 2021) 
10.1038/s41467-020-16525-w

Huan C, Kim D, Ou P, Alfonso A, and Stanek A. 2016. Mechanisms of interleukin-22's beneficial effects in acute pancreatitis. World J Gastrointest Pathophysiol 7:108-116. 10.4291/wjgp.v7.i1.108

Huang Y, Xiao M, Ou J, Lv Q, Wei Q, Chen Z, Wu J, Tu L, Jiang Y, Zhang X, Qi J, Qiu M, Cao S, and Gu J. 2020. Identification of the urine and serum metabolomics signature of gout. Rheumatology (Oxford) 59:29602969. 10.1093/rheumatology/keaa018

Kuwabara M, Kuwabara R, Hisatome I, Niwa K, Roncal-Jimenez CA, Bjornstad P, Andres-Hernando A, Sato Y, Jensen T, Garcia G, Ohno M, Hill JO, Lanaspa MA, and Johnson RJ. 2017. "Metabolically Healthy" Obesity and Hyperuricemia Increase Risk for Hypertension and Diabetes: 5-year Japanese Cohort Study. Obesity (Silver Spring) 25:1997-2008. 10.1002/oby.22000

Lamas B, Richard ML, Leducq V, Pham HP, Michel ML, Da Costa G, Bridonneau C, Jegou S, Hoffmann TW, Natividad JM, Brot L, Taleb S, Couturier-Maillard A, Nion-Larmurier I, Merabtene F, Seksik P, Bourrier A, Cosnes J, Ryffel B, Beaugerie L, Launay JM, Langella P, Xavier RJ, and Sokol H. 2016. CARD9 impacts colitis by altering gut microbiota metabolism of tryptophan into aryl hydrocarbon receptor ligands. Nat Med 22:598-605. 10.1038/nm.4102

Li F, Li X, Liu G, Gao C, and Li X. 2019. Bone Marrow Mesenchymal Stem Cells Decrease the Expression of RANKL in Collagen-Induced Arthritis Rats via Reducing the Levels of IL-22. J Immunol Res 2019:8459281. $10.1155 / 2019 / 8459281$

Lu DH, Guo XY, Qin SY, Luo W, Huang XL, Chen M, Wang JX, Ma SJ, Yang XW, and Jiang HX. 2015. Interleukin-22 ameliorates liver fibrogenesis by attenuating hepatic stellate cell activation and downregulating the levels of inflammatory cytokines. World J Gastroenterol 21:1531-1545. 10.3748/wjg.v21.i5.1531

Luo G, Yi T, Zhang G, Guo X, and Jiang X. 2017. Increased circulating Th22 cells in patients with acute gouty arthritis: A CONSORT-compliant article. Medicine (Baltimore) 96:e8329. 10.1097/MD.0000000000008329

Luo Y, Wang L, Liu XY, Chen X, Song YX, Li XH, Jiang C, Peng A, and Liu JY. 2018. Plasma profiling of amino acids distinguishes acute gout from asymptomatic hyperuricemia. Amino Acids 50:1539-1548. 10.1007/s00726018-2627-2

Marijnissen RJ, Koenders MI, Smeets RL, Stappers MH, Nickerson-Nutter C, Joosten LA, Boots AM, and van den Berg WB. 2011. Increased expression of interleukin-22 by synovial Th17 cells during late stages of murine experimental arthritis is controlled by interleukin-1 and enhances bone degradation. Arthritis Rheum 63:2939-2948. 10.1002/art.30469

Medrano M, Carrillo-Cruz E, Montero I, and Perez-Simon JA. 2018. Vitamin D: Effect on Haematopoiesis and Immune System and Clinical Applications. Int J Mol Sci 19. 10.3390/ijms19092663

Miyazaki Y, Nakayamada S, Kubo S, Nakano K, Iwata S, Miyagawa I, Ma X, Trimova G, Sakata K, and Tanaka Y. 2018. Th22 Cells Promote Osteoclast Differentiation via Production of IL-22 in Rheumatoid Arthritis. Front Immunol 9:2901. 10.3389/fimmu.2018.02901

Nakayama M. 2018. Macrophage Recognition of Crystals and Nanoparticles. Front Immunol 9:103. 10.3389/fimmu.2018.00103

Neogi T, Jansen TL, Dalbeth N, Fransen J, Schumacher HR, Berendsen D, Brown M, Choi H, Edwards NL, Janssens HJ, Liote F, Naden RP, Nuki G, Ogdie A, Perez-Ruiz F, Saag K, Singh JA, Sundy JS, Tausche AK, Vazquez-Mellado J, Yarows SA, and Taylor WJ. 2015. 2015 Gout Classification Criteria: an American College of Rheumatology/European League Against Rheumatism collaborative initiative. Arthritis Rheumatol

Peerf reviewing PDF | (2021:07:63744:2:1:NEW 3 Nov 2021) 
67:2557-2568. 10.1002/art.39254

Pepys MB, and Hirschfield GM. 2003. C-reactive protein: a critical update. J Clin Invest 111:1805-1812. 10.1172/JCl18921

Perusina Lanfranca M, Lin Y, Fang J, Zou W, and Frankel T. 2016. Biological and pathological activities of interleukin-22. J Mol Med (Berl) 94:523-534. 10.1007/s00109-016-1391-6

Qin JJ, Lu ZY, Jiao ZP, Zhu XJ, Wang YX, and Tang H. 2013. [Modified TRIzol method for RNA and DNA co-extraction from blood]. Fa Yi Xue Za Zhi 29:209-211.

Richette P, and Bardin T. 2010. Gout. Lancet 375:318-328. 10.1016/S0140-6736(09)60883-7

Salehzadeh F, Mohammadikebar Y, Haghi RN, Asl SH, and Enteshary A. 2019. Familial Mediterranean Fever Gene Mutations and Gout as an Auto-Inflammatory Arthropathy. Med Arch 73:55-57. 10.5455/medarh.2019.73.55-57

Singh JA, and Gaffo A. 2020. Gout epidemiology and comorbidities. Semin Arthritis Rheum 50:S11-S16. 10.1016/j.semarthrit.2020.04.008

Stack AG, Johnson ME, Blak B, Klein A, Carpenter L, Morlock R, Maguire AR, and Parsons VL. 2019. Gout and the risk of advanced chronic kidney disease in the UK health system: a national cohort study. BMJ Open 9:e031550. 10.1136/bmjopen-2019-031550

Teumer A, Li Y, Ghasemi S, Prins BP, Wuttke M, Hermle T, Giri A, Sieber KB, Qiu C, Kirsten H, Tin A, Chu AY, Bansal N, Feitosa MF, Wang L, Chai JF, Cocca M, Fuchsberger C, Gorski M, Hoppmann A, Horn K, Li M, Marten J, Noce D, Nutile T, Sedaghat S, Sveinbjornsson G, Tayo BO, van der Most PJ, Xu Y, Yu Z, Gerstner L, Arnlov J, Bakker SJL, Baptista D, Biggs ML, Boerwinkle E, Brenner H, Burkhardt R, Carroll RJ, Chee ML, Chee ML, Chen M, Cheng CY, Cook JP, Coresh J, Corre T, Danesh J, de Borst MH, De Grandi A, de Mutsert R, de Vries APJ, Degenhardt F, Dittrich K, Divers J, Eckardt KU, Ehret G, Endlich K, Felix JF, Franco OH, Franke A, Freedman BI, Freitag-Wolf S, Gansevoort RT, Giedraitis V, Gogele M, Grundner-Culemann F, Gudbjartsson DF, Gudnason V, Hamet P, Harris TB, Hicks AA, Holm H, Foo VHX, Hwang SJ, Ikram MA, Ingelsson E, Jaddoe VWV, Jakobsdottir J, Josyula NS, Jung B, Kahonen M, Khor CC, Kiess W, Koenig W, Korner A, Kovacs P, Kramer H, Kramer BK, Kronenberg F, Lange LA, Langefeld CD, Lee JJ, Lehtimaki T, Lieb W, Lim SC, Lind L, Lindgren CM, Liu J, Loeffler M, Lyytikainen LP, Mahajan A, Maranville JC, Mascalzoni D, McMullen B, Meisinger C, Meitinger T, Miliku K, Mook-Kanamori DO, Muller-Nurasyid M, Mychaleckyj JC, Nauck M, Nikus K, Ning B, Noordam R, Connell JO, Olafsson I, Palmer ND, Peters A, Podgornaia AI, Ponte B, Poulain T, Pramstaller PP, Rabelink TJ, Raffield LM, Reilly DF, Rettig R, Rheinberger M, Rice KM, Rivadeneira F, Runz H, Ryan KA, Sabanayagam C, Saum KU, Schottker B, Shaffer CM, Shi Y, Smith AV, Strauch K, Stumvoll M, Sun BB, Szymczak S, Tai ES, Tan NYQ, Taylor KD, Teren A, Tham YC, Thiery J, Thio CHL, Thomsen H, Thorsteinsdottir U, Tonjes A, Tremblay J, Uitterlinden AG, van der Harst P, Verweij N, Vogelezang S, Volker $\mathrm{U}$, Waldenberger $\mathrm{M}$, Wang $\mathrm{C}$, Wilson OD, Wong C, Wong TY, Yang $\mathrm{Q}$, Yasuda M, Akilesh S, Bochud $\mathrm{M}$, Boger CA, Devuyst O, Edwards TL, Ho K, Morris AP, Parsa A, Pendergrass SA, Psaty BM, Rotter JI, Stefansson K, Wilson JG, Susztak K, Snieder H, Heid IM, Scholz M, Butterworth AS, Hung AM, Pattaro C, and Kottgen A. 2019. Genome-wide association meta-analyses and fine-mapping elucidate pathways influencing albuminuria. Nat Commun 10:4130. 10.1038/s41467-019-11576-0

Wallace SL, Robinson H, Masi AT, Decker JL, McCarty DJ, and Yu TF. 1977. Preliminary criteria for the classification of the acute arthritis of primary gout. Arthritis Rheum 20:895-900. 10.1002/art.1780200320

Wen H, Luo J, Zhang X, Zhang C, Zhao X, Wang X, and Li X. 2015. [1,25(OH)2-Vitamin-D3 attenuates Th17-related 
467

468

469

470

471

472

473

474

475

476

477

478

479

480

481

482

483

484

485 cytokines expression in peripheral blood mononuclear cells in patients with early-diagnosed rheumatoid arthritis]. Zhonghua Nei Ke Za Zhi 54:317-321.

Xue M, Zhao J, Ying L, Fu F, Li L, Ma Y, Shi H, Zhang J, Feng L, and Liu P. 2017. IL-22 suppresses the infection of porcine enteric coronaviruses and rotavirus by activating STAT3 signal pathway. Antiviral Res 142:68-75. 10.1016/j.antiviral.2017.03.006

Yang M, Yang BO, Gan H, Li X, Xu J, Yu J, Gao L, and Li F. 2015. Anti-inflammatory effect of 1,25-dihydroxyvitamin D3 is associated with crosstalk between signal transducer and activator of transcription 5 and the vitamin D receptor in human monocytes. Exp Ther Med 9:1739-1744. 10.3892/etm.2015.2321

Yang X, and Zheng SG. 2014. Interleukin-22: a likely target for treatment of autoimmune diseases. Autoimmun Rev 13:615-620. 10.1016/j.autrev.2013.11.008

Yin C, Liu B, Li Y, Li X, Wang J, Chen R, Tai Y, Shou Q, Wang P, Shao X, Liang Y, Zhou H, Mi W, Fang J, and Liu B. 2020. IL-33/ST2 induces neutrophil-dependent reactive oxygen species production and mediates gout pain. Theranostics 10:12189-12203. 10.7150/thno.48028

Zhang YG, Lu R, Wu S, Chatterjee I, Zhou D, Xia Y, and Sun J. 2020. Vitamin D Receptor Protects Against Dysbiosis and Tumorigenesis via the JAK/STAT Pathway in Intestine. Cell Mol Gastroenterol Hepatol 10:729-746. 10.1016/j.jcmgh.2020.05.010

Zhu W, Deng Y, and Zhou X. 2018. Multiple Membrane Transporters and Some Immune Regulatory Genes are Major Genetic Factors to Gout. Open Rheumatol J 12:94-113. 10.2174/1874312901812010094 
Figure 1

mRNA and protein levels of IL-22, VD-R and $1,25(\mathrm{OH})_{2} \mathrm{VD} 3$ in the plasma from gouty arthritis patients

$\mathbf{A}$

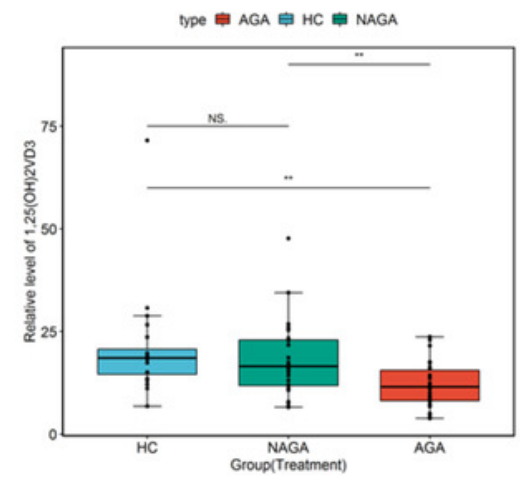

C

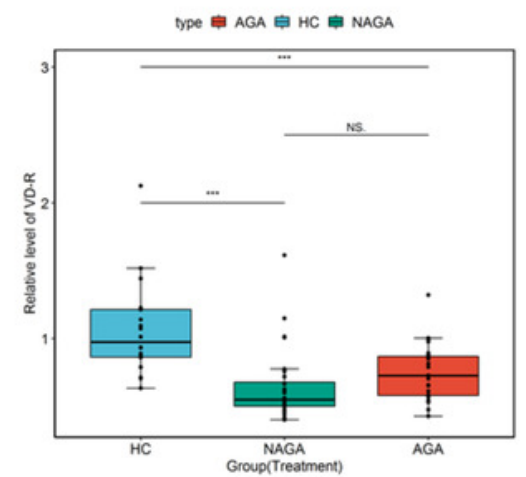

B

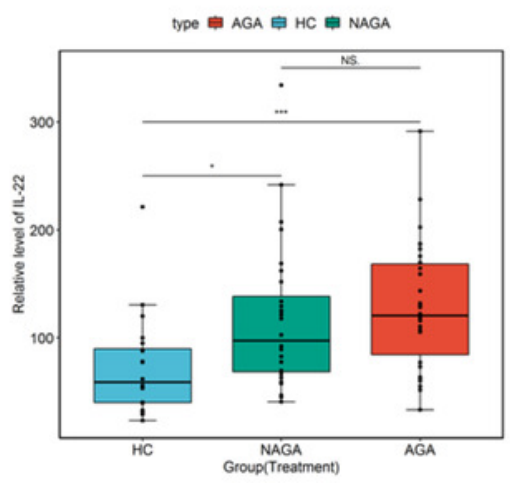

D

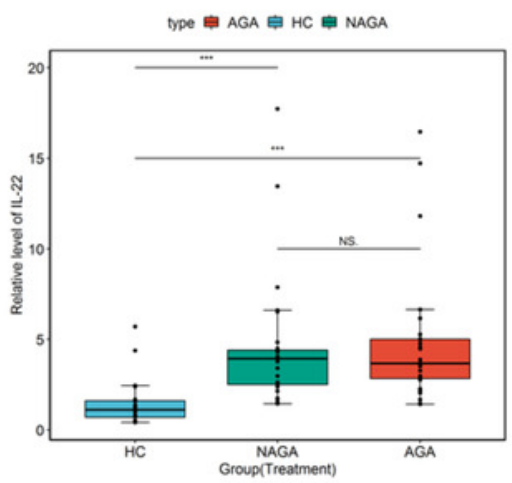


Figure 2

Metabolomic landscape of the GA patients

$\mathbf{A}$
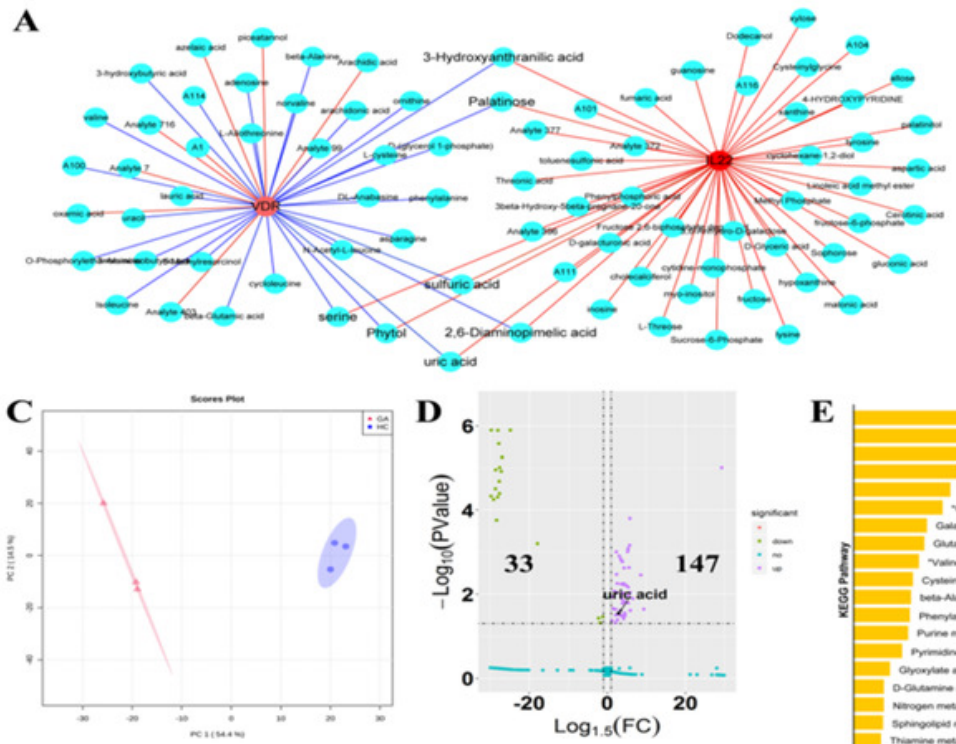

$D_{6}$

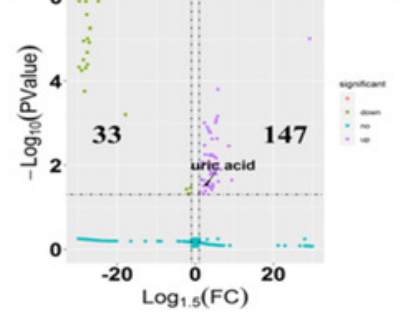

B

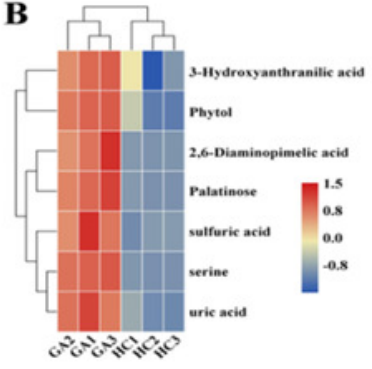

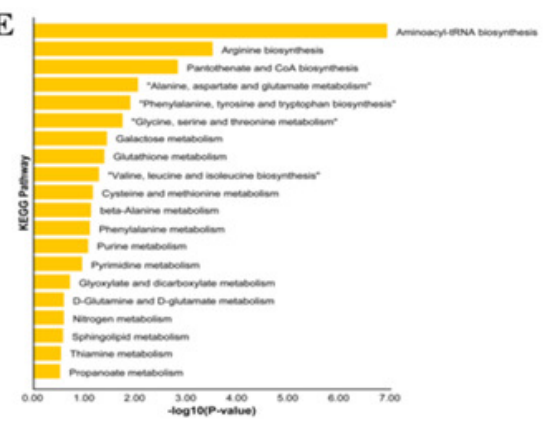


Figure 3

Correlations in the serum levels of LogCRP and IL-22 or $1,25(\mathrm{OH})_{2} \mathrm{VD} 3$

A

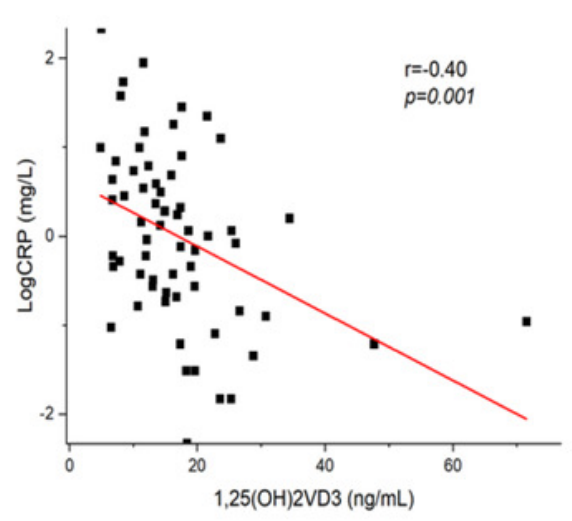

B

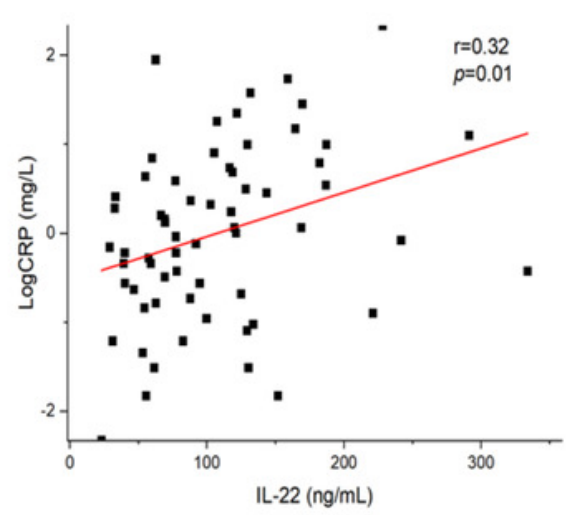


Figure 4

Complex regulation network and imperative roles of IL-22 and VD-R were involved in GA-related inflammatory responses

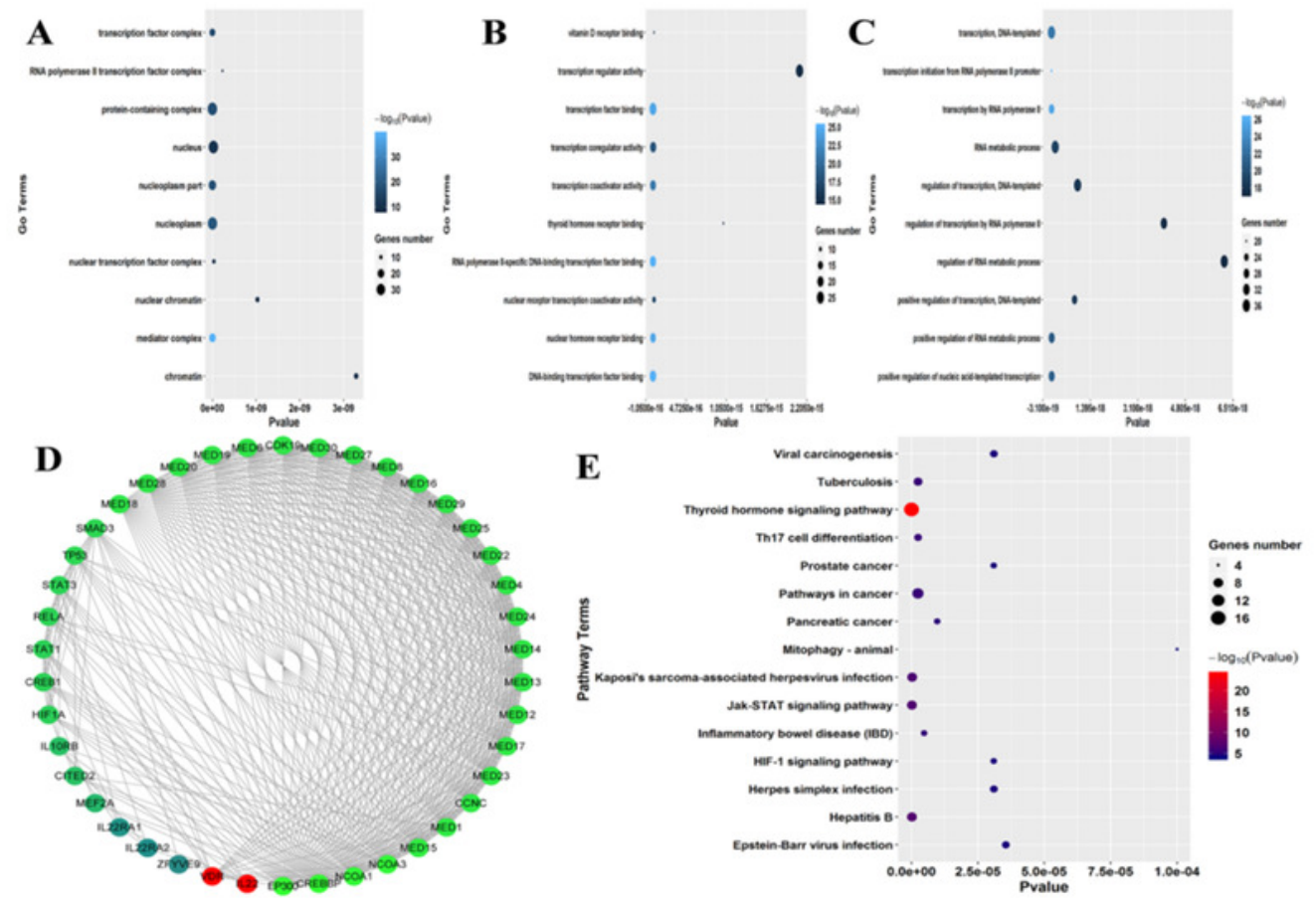


Table $\mathbf{1}$ (on next page)

Patient characteristics 


\begin{tabular}{|c|c|c|c|c|}
\hline \multirow{3}{*}{ Variable } & AGA Patients & NAGA Patients & $\mathrm{HC}$ & \multirow[t]{3}{*}{$p$} \\
\hline & & & & \\
\hline & $\mathrm{N}=30$ & $\mathrm{~N}=28$ & $\mathrm{~N}=20$ & \\
\hline Age,y & $43 \pm 15.68$ & $36.7 \pm 11.4$ & $43.0 \pm 8.6$ & \\
\hline BMI kg/m²\# & $26.6 \pm 3.5$ & $27.4 \pm 4.5$ & $24.23 \pm 1.3$ & $*=0.022, \#=0.003$ \\
\hline UA umol/L* & $446.63 \pm 150.95$ & $419.14 \pm 149.20$ & $341.45 \pm 66.99$ & $*=0.08$ \\
\hline $\mathrm{Cr}$ umol/L*\# & $100.72 \pm 26.33$ & $92.58 \pm 19.34$ & $79.72 \pm 13.19$ & $*=0.001, \#=0.041$ \\
\hline Platelet $10^{\wedge 9} / \mathrm{L}$ & $218.83 \pm 63.97$ & $250.43 \pm 71.45$ & $227.15 \pm 54.68$ & \\
\hline $\mathrm{ESR} \mathrm{mm} / \mathrm{h}$ & $20.2 \pm 5.2$ & $13.60 \pm 4.8$ & $10.8 \pm 3.5$ & \\
\hline $\mathrm{CRP} \mathrm{mg} / \mathrm{L} * \triangle$ & $24.42 \pm 13.42$ & $2.45 \pm 1.50$ & $1.85 \pm 1.68$ & $*<0.001, \triangle<0.001$ \\
\hline Cholesterin $\mathrm{mmol} / \mathrm{L}$ & $4.30 \pm 0.83$ & $4.57 \pm 0.98$ & $4.23 \pm 0.6$ & \\
\hline Triglyceride $\mathrm{mmol} / \mathrm{L}$ & $1.97 \pm 0.96$ & $2.45 \pm 1.5$ & $1.85 \pm 1.68$ & \\
\hline Blood glucose $\mathrm{mmol} / \mathrm{L}^{*}$ & $5.64 \pm 1.06$ & $5.35 \pm 0.48$ & $5.03 \pm 0.51$ & $*<0.011$ \\
\hline Tophi $n$ & $5(16.7 \%)$ & $3(10.7 \%)$ & Not available & \\
\hline Disease duration $\mathrm{m}$ & $27.8 \pm 34.5$ & $41.9 \pm 52.5$ & Not available & \\
\hline \multicolumn{5}{|l|}{ Comorbidities } \\
\hline Hypertension & $7(24.1 \%)$ & $7(25.0 \%)$ & $1(5 \%)$ & \\
\hline Diabetes & $4(13.3 \%)$ & $5(17.9 \%)$ & $0(0.0 \%)$ & \\
\hline
\end{tabular}

2 AGA: acute gouty; BMI: body mass index; CRP: C-reactive protein;

3 ESR: erythrocyte sedimentation rate; HC: healthy controls; NAGA: non-acute gouty arthritis; UA: uric acid; 
$4 * p<0.05$, between AGA patients vs. HC. \# $p<0.05$, between NAGA patients vs. HC. $\triangle p<0.05$, between

$5 \quad$ NAGA patients vs. AGA patients;

6 
Table 2 (on next page)

Pathway analysis on metabolites closely associated with IL22 and VDR 


\begin{tabular}{cccc}
\hline name & Hits & Raw p & Impact \\
\hline Aminoacyl-tRNA biosynthesis & 9 & $1.64 \mathrm{E}-05$ & 0.16667 \\
Pantothenate and CoA biosynthesis & 5 & 0.000285 & 0.02143 \\
Purine metabolism & 7 & 0.004921 & 0.03226 \\
Phenylalanine, tyrosine and tryptophan & 2 & 0.006345 & 1 \\
biosynthesis & 3 & 0.009984 & 0.06091 \\
Arginine biosynthesis & 2 & 0.027162 & 0 \\
Valine, leucine and isoleucine biosynthesis & 3 & 0.030917 & 0.39925 \\
beta-Alanine metabolism & 2 & 0.04182 & 0.35714 \\
Phenylalanine metabolism & & &
\end{tabular}

1 